Rev. salud pública. 10 (1):178-188, 2008

\title{
Modelos empleados para la Toma de Decisiones en el Cuidado de la Salud
}

\author{
Models used for decision-making in health care
}

\author{
Ricardo Sánchez-Pedrazaํㅡㄹ Oscar Gamboa² y Jorge A. Díaz ${ }^{3}$
}

\begin{abstract}
1 Facultad de Medicina, Universidad Nacional de Colombia. rsanchezpe@unal.edu.co
2 Instituto Nacional de Cancerologia. oagamboag@unal.edu.co

3 Facultad de Ciencias, Departamento de Farmacia, Universidad Nacional de Colombia.

jadiazr@unal.edu.co
\end{abstract}

Recibido 27 Marzo 2007/Enviado para Modificación 8 Diciembre 2007/Aceptado 7 Enero 2008

\section{RESUMEN}

El análisis de decisiones es un grupo de herramientas que permiten apoyar y manejar un proceso de evaluación estructurado. Esta metodología se usa ampliamente en la evaluación económica para planeación o programas de salud. Este artículo delinea algunas características de las decisiones complejas y muestra los fundamentos y etapas que deben considerarse cuando se toman decisiones en un escenario de incertidumbre (definición del problema, selección de un marco temporal de análisis adecuado, estructuración del problema, desarrollo de un modelo para análisis, selección de la mejor alternativa y realización de análisis de sensibilidad). Finalmente se presentan algunas críticas que se han hecho a esta metodología.

Palabras Clave: Toma de decisiones asistida por computador, toma de decisiones , técnicas de apoyo para la decisión, cadenas de Markov, atención a la salud (fuente: DeCS, BIREME).

\section{ABSTRACT}

Decision analysis consists of a set of tools supporting and handling structured evaluation. Such methodology is widely used for the economic evaluation of health care planning and programmes. This article outlines some characteristics regarding complex decision-making and shows the fundamental issues and stages considered when making decisions in an uncertain scenario (problem definition, choosing an appropriate time-frame, structuring the problem, developing a model for analysing it, selecting the best alternative and analysing sensitivity). Some criticism of this decision-making method is then made.

Key Words: Decision-making, computer-assisted decision-making, decision support techniques, Markov chains, public health (source: DeCS, BIREME). 
$\mathrm{T}$ omar una decisión implica escoger entre varias alternativas. Tomar la mejor decisión supone haber hecho un análisis de lo que hubiera sucedido si cada una de las posibles alternativas se hubiera seleccionado. La toma de decisiones es un acto cotidiano que está involucrado en múltiples actividades que generalmente se hace por técnicas como la adivinanza, la reacción visceral, la intuición, o la experiencia basada en opiniones o sucesos muy parecidos.

La toma decisiones de forma intuitiva generalmente resulta poco eficiente dado que esta estrategia no suele incorporar todos los factores que pueden afectar la decisión y sus resultados. Pocas decisiones se toman con plena certidumbre sobre sus posibles consecuencias. El proceso de tomar decisiones puede ser mejorado utilizando una metodología que combina una estructura explícita y una técnica cuantitativa de análisis y que se ha denominado "enfoque sistemático de toma de decisiones bajo condiciones de incertidumbre"(1). Tal metodología, que no es nativa del área de la salud, se concibió inicialmente como un proceso iterativo para generar mayor conocimiento y facilitar la generación de alternativas creativas que ayudaran a los tomadores de decisiones a realizar mejores decisiones (2).

¿Por qué puede ser difícil tomar una decisión?

La dificultad para tomar una decisión se relaciona con tres aspectos: Estructurales, personales y políticos (3):

1. Aspectos estructurales: Dentro de esta categoría se agrupan las siguientes situaciones:

a. El grado de incertidumbre: La incertidumbre se presenta cuando no se conoce completamente la probabilidad de que ocurra un evento. Al existir incertidumbre resulta imposible conocer de antemano cuál va a ser el resultado de una decisión. La incertidumbre puede relacionarse con el diagnóstico, la exactitud de las herramientas diagnósticas, la calidad y disponibilidad de las fuentes de información, la historia natural de las enfermedades, las opciones que tomen los pacientes y los resultados del tratamiento. Al existir múltiples fuentes de incertidumbre se hace más complicado tener un esquema claro de las diferentes opciones y resultados de las posibles alternativas de decisión.

b. Cantidad de alternativas disponibles: Entre mayor sea el número de alternativas entre las cuales haya que escoger, mayor será la complejidad del recorrido entre la decisión y las posibles consecuencias. La utilización de herramientas gráficas, como los árboles de decisión, permiten tener una visión 
más clara y precisa de los diferentes cursos que pueden tomar las múltiples alternativas de decisión.

c. Consecuencias de tomar la decisión: Entre más graves son las consecuencias de tomar una decisión incorrecta, más difícil resulta optar por una alternativa. d. Frecuencia con la que se toman decisiones parecidas: A menor frecuencia, mayor suele ser la dificultad para tomar la decisión.

2. Aspectos personales: Algunas características psicológicas de quien toma la decisión pueden dificultar este proceso. Por ejemplo los patrones de personalidad obsesivos, caracterizados por el perfeccionismo, la rigurosidad y la preocupación exagerada por detalles, suelen presentar una tendencia a complicar la toma de decisiones. Por otro lado las personas impulsivas tienden a tomar más fácilmente decisiones, pero de manera no acertada, dada la falta de análisis que aplican a su conducta. El tomador de decisiones debería ser una persona que tenga claridad sobre las características personales que pudieran afectar el proceso de seleccionar la alternativa acertada.

3. Aspectos políticos: En algunos casos la alternativa más acertada, escogida mediante un proceso racional y sistematizado, debe supeditarse a consideraciones de orden político que resultan prioritarias.

Para el manejo de los aspectos estructurales se ha desarrollado una metodología que permite utilizar un abordaje cuantitativo y estructurado de las situaciones de toma de decisiones, y que permite evaluar decisiones que se deben tomar en situaciones en las que se presenten alternativas complejas y diversas fuentes de incertidumbre. Dicha metodología, que como ya se mencionó se ha llamado "análisis de decisiones bajo condiciones de incertidumbre", permite:

1. Generar una estructura gráfica que permita ver claramente la relación entre alternativas y consecuencias.

2. Asignar valores a las fuentes de incertidumbre: Asignando valores de probabilidad a los puntos de incertidumbre se facilita su manejo (4). Si hay certidumbre sobre un evento, su probabilidad de ocurrir es de uno (obviamente la probabilidad de que no ocurra es cero). Si no hay certidumbre, la probabilidad de ocurrir será menor que uno. Posteriormente se mostrará cómo se asignan estos valores de probabilidad.

3. Facilitar la comparación entre las diferentes alternativas en términos numéricos. En esta metodología, a las alternativas posibles se les asigna un valor numérico que corresponde a un concepto estadístico denominado "valor esperado".En estadística el valor esperado (o esperanza matemática) de una 
variable aleatoria es la suma de la probabilidad de cada suceso multiplicada por su valor. Dicho de otra forma el valor esperado se asemeja al resultado que se espera en promedio si se repitiera un experimento (como lanzar una moneda, o medir la talla de un grupo de personas, por ejemplo) muchas veces. La utilidad del valor esperado es permitir elegir entre distintas alternativas. En general la alternativa que se elige es aquella que tenga el valor esperado más alto (años de vida ganados, muertes evitadas, por ejemplo), pero en ocasiones se selecciona aquella con el valor más bajo (mortalidad, costos, por ejemplo) (5).

Con estos insumos el tomador de decisiones puede identificar fácilmente las opciones viables, predecir sus consecuencias o desenlaces, valorar la probabilidad de los posibles resultados, determinar el valor de cada uno de los desenlaces, y seleccionar la decisión que puede proveer el mejor resultado.

En el área del cuidado de la salud, el análisis de decisiones es una metodología que se desarrolló alrededor de situaciones clínicas en pacientes individuales. Sin embargo, se usa cada vez más frecuentemente en análisis económicos y para la toma de decisiones políticas $(6,7)$.

Etapas en un análisis de decisiones

Dado que el análisis de decisiones es una metodología que pretende, no solo facilitar, sino además sistematizar el proceso de selección de alternativas, para hacerlo, de alguna manera replicable, se ha definido una estructuración a lo largo de una serie de etapas sucesivas (8) (debe tenerse en cuenta que la replicabilidad de los resultados se ve fuertemente afectada por los aspectos personales y políticos mencionados atrás):

1. Definición del problema:

Un problema se ha definido como la distancia que hay entre una situación que existe y otra que se prefiere. El primer paso suele ser escribir el problema utilizando pocas palabras, preferiblemente recurriendo al uso de verbos en infinitivo, y hacer una descripción de ciertos detalles como magnitud, posibles causas o responsables, momento de inicio, identificación de afectados con el problema, estrategias con las que ha intentado solucionarse, y posible resultado si no se intenta ninguna solución. Este primer paso puede ser suficiente para tomar una decisión acertada, lo cual sucede cuando se vislumbran alternativas de decisión que no se habían considerado, cuando se evidencia que se estaba sobredimensionando la gravedad del problema, cuando se ve que se estaba tratando de solucionar el problema equivocado (esto se ha denominado "error 
tipo tres"), o incluso cuando se encuentra que si no se hace nada, no pasa nada, y lo que genera la dificultad muchas veces son los intentos de solución (en este último caso se habla de pseudo problemas). Otra posibilidad que surge en este primer paso es encontrar que el problema tiene sub problemas, lo cual implica efectuar un trabajo de jerarquización y definir claramente cuál es el problema primario o principal (9).

2. Definición del horizonte de análisis (marco temporal):

Cuando se evalúan alternativas relacionadas con patologías crónicas, el horizonte de análisis suele ser largo. En estos casos los eventos pueden reaparecer durante el período definido en el marco temporal: esto supone utilizar estrategias especiales de análisis (cadenas y modelos de Markov para eventos recurrentes). Por otro lado, la evaluación de alternativas relacionadas con complicaciones a corto plazo o con eventos agudos supone un horizonte de análisis corto. En otras palabras, el horizonte de análisis dependerá de la naturaleza del problema de salud que se esté evaluando. Cualquiera que sea el caso, debe hacerse una especie de negociación entre la exactitud del modelo analítico y la simplicidad del mismo (10). Un modelo no debe ser una representación completa del mundo real sino una síntesis de sus componentes más importantes (11).

3. Estructuración del problema:

La etapa de estructuración supone seguir esta secuencia:

- Identificar las alternativas relevantes: Una vez identificado el problema, deben plantearse una serie de alternativas, las cuales suelen caer en alguna de las siguientes categorías:

- Esperar y observar, lo cual es equivalente a la política de "no hacer nada".

- Iniciar una intervención.

- Buscar más información antes de decidir una intervención.

- Definir las consecuencias de cada alternativa identificada previamente: Cada alternativa genera una serie de consecuencias cuya incorporación en la estructuración del problema supone que esté suficientemente sustentada con algún tipo de evidencia. Las consecuencias pueden categorizarse en dos grupos: i) consecuencias intermedias. ii) desenlaces finales. Por ejemplo, si un médico se encuentra prestando su servicio rural en un lugar apartado donde no dispone de medios diagnósticos ni posibilidades de remitir rápidamente a sus pacientes, y recibe a un paciente con una herida por arma de fuego en el cráneo, podría 
plantearse, de manera general, que hay dos alternativas: esperar y observar, o arriesgarse a intervenir quirúrgicamente al paciente. Las consecuencias de cada alternativa podrían definirse de manera simplista en dos categorías: el paciente sobrevive o muere (aquí las consecuencias se presentan directamente como los desenlaces finales). Sin embargo, considerando la falta de pericia quirúrgica del médico, puede plantearse una consecuencia intermedia de la alternativa intervencionista: que la cirugía se complique o que no se complique; cada una de estas consecuencias intermedias puede resultar en la salvación o en la muerte del paciente. En la Figura 1 se muestra esta secuencia de consecuencias, con unas convenciones de representación gráfica que se explicarán más adelante.

Figura 1. Dos modelos de definición de consecuencias de las alternativas
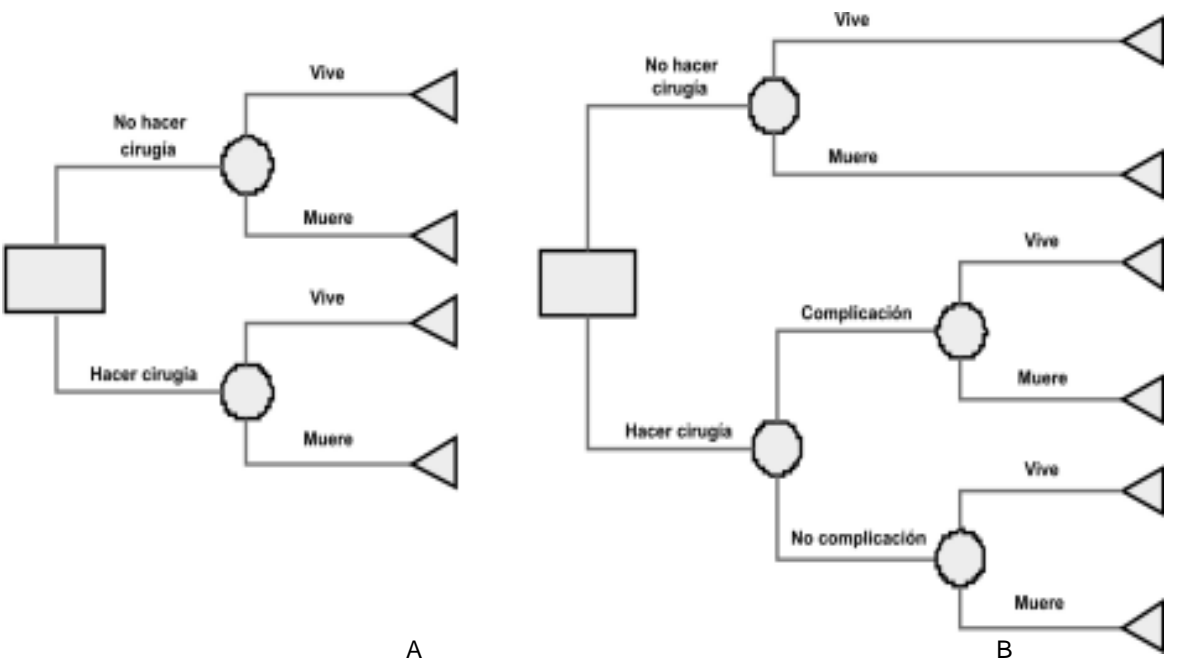

A. Consecuencias como desenlaces finales.

B. Consecuencias considerando opciones intermedias antes de los desenlaces finales.

- Establecer la probabilidad con la que pueden suceder las diferentes consecuencias de las alternativas planteadas: Un paso fundamental en este tipo de análisis es cuantificar la incertidumbre: esto se logra asignando valores de probabilidad a cada una de las consecuencias emanadas de las alternativas propuestas para la solución del problema.

- Asignar un valor a los desenlaces: Un desenlace es lo que finalmente sucede si se escoge una de las alternativas planteadas. En esta fase de la estructuración del problema, se debe asignar un valor numérico a los desenlaces. Esta 
calificación se realiza con base en el concepto de utilidad. De forma práctica la utilidad se puede considerar como la medida de la preferencia de las personas por un resultado bien o servicio. En particular, en el campo sanitario, seria la medida cuantitativa de la preferencia de las personas por un resultado específico en salud (12).

4. Desarrollo de un modelo del problema:

Un modelo es una abstracción o representación de un sistema real, de una idea o de un objeto (13). En un análisis de decisiones el modelo es una estructura que busca representar la realidad del problema, combinando los insumos generados en la etapa previa (etapa de estructuración), con el propósito de efectuar una serie de cálculos matemáticos que ayuden a tomar la decisión. Los modelos más utilizados como estrategia de análisis son los árboles de decisión (14). Mediante su uso se pueden integrar, de manera secuencial, los diferentes elementos que surgen una vez se ha estructurado el problema. En la construcción de un árbol de decisión se siguen las siguientes convenciones (Figura 2):

Punto de arranque: Es la presentación del problema en pocas palabras.

Nodo de decisión: Representa un punto donde se toma una conducta, como por ejemplo las diferentes alternativas de solución que se plantean al problema. Su forma de representación en el árbol es un cuadrado.

Nodo probabilístico o de azar: Muestra el punto donde se generan las diferentes consecuencias de una decisión, que no están bajo el control de quien toma las decisiones. Como previamente se comentó, las consecuencias generadas de un nodo de azar tienen un componente de incertidumbre cuantificable por medio de probabilidades. Se representan gráficamente con un círculo.

Nodo terminal: Representa los desenlaces finales. Se representa con un triángulo.

Conectores: Son líneas que unen los diferentes elementos descritos previamente. La manera de conectar las diferentes estructuras obedece a una secuencia en la que, lo que está a la izquierda, temporalmente ha ocurrido antes que lo que está a la derecha. La amplitud con la que se desarrolle el árbol corresponde al horizonte de análisis. 
Definiciones y valores: Los conectores que salen de un nodo de azar tienen en su parte superior la descripción del desenlace y el su parte inferior la cuantificación de la incertidumbre (valores de probabilidad). En el extremo de los nodos terminales se ubican los valores asignados a los desenlaces (utilidades).

Figura 2. Convenciones utilizadas en la construcción de un árbol de decisión

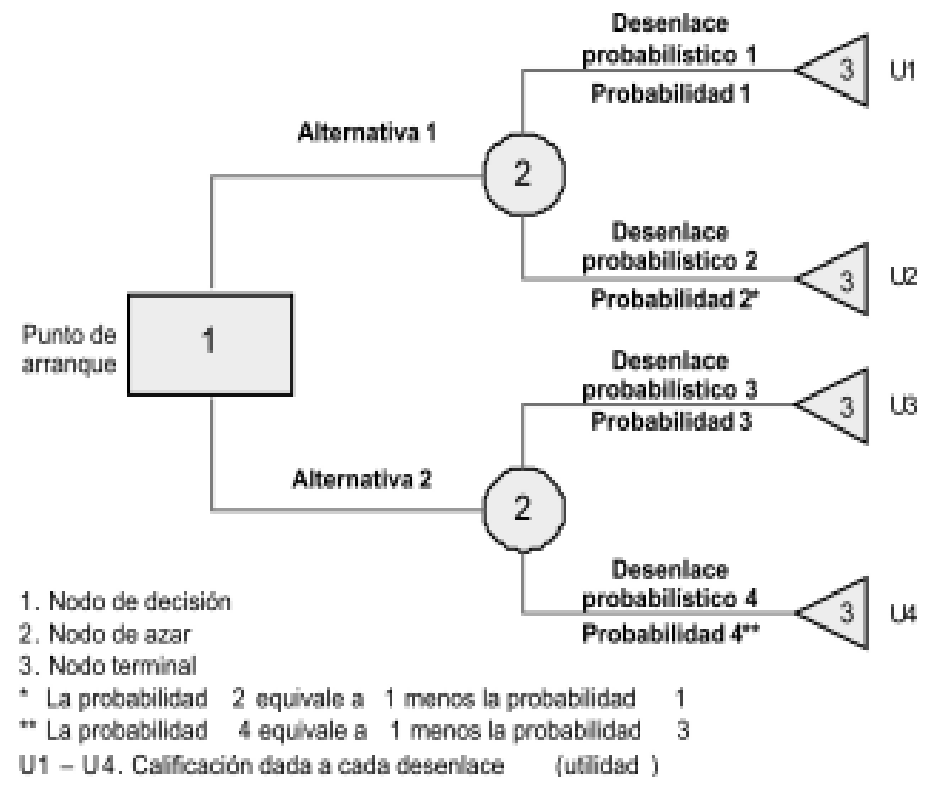

5. Efectuar análisis de sensibilidad:

Teniendo en cuenta que, ni los valores de probabilidad con que se mide la incertidumbre ni los valores asignados a los desenlaces son valores fijos, al efectuar un análisis de decisiones surge la duda sobre la posibilidad de que la solución al problema cambiara si los valores de la medición de incertidumbre y consecuencias variaran. Para resolver esta duda se realizan los análisis de sensibilidad. Ellos permiten evaluar los resultados ante la posible gama de valores que podrían tomar uno o más de los parámetros introducidos en el modelo. Como resultado se puede conocer la estabilidad de la conclusión dada la variabilidad de los supuestos introducidos en el modelo. Es frecuente que para estos análisis se utilicen herramientas gráficas. En los casos en los que la variabilidad se genera en un solo punto del modelo se utilizan los análisis de sensibilidad de un parámetro. Si la fuente de variabilidad proviene de varios puntos se habla de análisis de sensibilidad de múltiples parámetros. 
6. Selección de la "mejor" alternativa:

Al finalizar el análisis se obtendrán una serie de valores numéricos (valores esperados) que ponderarán de manera diferente las distintas alternativas de decisión. Dependiendo de la medida de utilidad manejada, se seleccionará la mejor opción (por ejemplo si se manejan años de vida saludable ganados, la mejor opción será aquella con el mayor valor). Sin embargo, hay que tener en cuenta que los resultados numéricos no son la única herramienta a la hora de tomar la decisión final. Existen casos en los cuales tiene más peso algún otro tipo de factor, como pueden ser aspectos clínicos o políticos. La recomendación general al utilizar este tipo de métodos es que son solo herramientas para ayudar a decidir, mas no el único elemento que se toma en cuenta para la toma final de la decisión.

Críticas al análisis de decisiones

Los detractores de esta metodología aducen tres razones para no recomendar su uso (15):

\section{Dificultad}

Así existan herramientas manejables con computadores, la gran cantidad de opciones derivada de la toma de una decisión compleja, hace que la estructura a analizar se vuelva demasiado enmarañada y alejada de la realidad. Algunos teóricos de la economía, conscientes de la limitación impuesta por la complejidad que pueden alcanzar los modelos, han planteado que no es posible escoger la mejor opción. Dentro de esta tendencia se plantea también la utilización de modelos parsimoniosos que, si bien no capturan la integridad de la complejidad de la situación, incorporan los aspectos más relevantes de la misma. Los árboles de decisión tradicionales, presentan inconvenientes cuando se quiere representar eventos que pueden ocurrir más de una vez, eventos cuyas probabilidades de ocurrencia cambian con el tiempo, eventos que no ocurren inmediatamente y eventos que tienen implicaciones a largo plazo en los pacientes $(16,17)$. Las cadenas y modelos de Markov permiten manejar estas dificultades que se presentan con los árboles de decisión tradicionales, y son útiles cuando se quiere modelar problemas que involucran riesgos que dependen del tiempo.

\section{Falta de sensibilidad política}

Se ha planteado que esta metodología se engolosina con la estructura del problema pero que no le da énfasis a las dificultades que implica poner en la práctica la toma de la decisión. Aunque la objetividad y la neutralidad de valores se han adjudicado a esta metodología como una de sus fortalezas, se ha 
encontrado que las personas que no han participado en la toma de la decisión pueden mostrar resistencia ante el proceso de implementar la decisión. Esto ha podido verse en el poco impacto que han tenido herramientas para tomar decisiones, como las guías de práctica clínica o recomendaciones generadas en consensos.

\section{Limitación del razonamiento humano}

Esta es una consideración más de índole filosófica y se basa en el planteamiento de que no hay verdades absolutas, y que lo que se acepta como conocimiento no es más que el resultado de cómo una cultura ha preparado y aleccionado a un individuo para que evalúe y juzgue una situación. Desde esta perspectiva, los hechos sobre los que se basa un análisis de decisiones no son completamente objetivos, sino que están matizados por particularidades y características de un grupo cultural: así las cosas, no tendría mucho sentido efectuar un riguroso análisis numérico a unos datos que no son más que distorsiones introducidas por la cultura

\section{REFERENCIAS}

1. Bootman J, Townsend R, McGhan W. Principles of Pharmacoeconomics, 1991, Harvey Whitney Books Company, Cincinnati, OH. Pp. 112-116.

2. Howard RA. Decision analysis: Practice and promise. Management Science 1988;34:67995.

3. Skinner DC. Introduction to Decisión Analysis. 2nd edition. Gainesville: Probabilistic Publishing; 1995. p. 142-150.

4. Gross R. Making Medical decisions. An Approach to Clinical Cecision Making for Practicing Physicians. Philadelphia: American College of Physicians; 1999. p. 20.

5. Sox. H, Blatt M, Higgins M, Marton K. Medical Decisión Making, 1988 Butterworh publishers Stoneham, MA. Pp. 147-158.

6. Petitti DB. Meta-analysis, decision analysis and cost-effectiveness analysis. Methods for quantitative synthesis in medicine. 2nd edition. New Methods for quantitative synthesis in medicine. 2nd edition. New York: Oxford University Press;2000. p. 17.

7. Murphy JM, Berwick DM, Weinstein MC, Borus JF, Budman SH, Klerman GL. Performance of screening tests: Application of roc analysis. Arch Gen Psychiatry 1987;44:550-555.

8. Sánchez R, Echeverri J.Análisis de decisiones. Una herramienta para la práctica clínica. En: Ardila E, Sánchez R, Echeverri J. Estrategias de Investigación en Medicina Clínica. Bogotá: Editorial Manual Moderno: 2001. 
9. Clemen RT. Making hard decisions. An introduction to decision analysis. 2nd edition. Pacific Grove: Duxbury Press;1996. p. 292-4.

10. Moskowltz AJ, Dunn VH, Lau J, Pauker SG. Can "hypersimplied" decision trees be used instead of Markov models? Med Decis Making. 1984;4;530.

11. Detsky AS, Naglie G, Krahn MD, Naimark D, Redelmeier DA. Primer on medical decision analysis: Part 1. Getting started. Med Decis Making. 1997;17(2):123-5.

12. Drummond M, O’brien B, Stoddart G, Torrance G. Métodos para la Evaluación económica de los Programas de Asistencia Sanitaria. $2^{a}$. Edición. Madrid, España: Ediciones Diaz Santos; 2001.pp. 161- 169.

13. Evans JR, Olson DL. Introduction to simulation and risk analysis. Upper Saddle River: Prentice Hall; 1998. p. 80-106.

14. Fineberg HV. Decision trees: construction, uses and limits. Bull Cancer (Paris) 1980;67:395-404

15. Golub AL. Decision Analysis. An integrated approach. New York: John Wiley \& Sons, Inc.;1997.pp. 12-22.

16. Briggs A, Sculpher M. An Introduction to Markov Modelling for Economic Evaluation. Pharmacoeconomics 1998; 13(4): 397-409.

17. Naimark D, Murray DK, Naglie G, Redelmeier D, Detsky AD. Primer on medical decision analysis: Part 5- working with Markov processes. Med Decis Making 1997; 17: 152-159. 